\title{
Children with lymphoma presenting with hemophagocytic lymphohistiocytosis
}

\author{
Tekin $\mathrm{Aksu}^{1 \oplus}$, Turan Bayhan ${ }^{2 \oplus}$, Belgin Gülhan ${ }^{3 \oplus}$, Selma Çakmakc1 ${ }^{2 \oplus}$, \\ Ayşe Selcen Oğuz ${ }^{4 \oplus}$, Neşe Yaralı1ø, Namık Yaşar Özbek ${ }^{1 \oplus}$, İnci İlhan ${ }^{2 \oplus}$ \\ ${ }^{1}$ Department of Pediatric Hematology and Oncology, University of Health Sciences, Ankara Child Health and Diseases Hematology \\ Oncology Training and Research Hospital; ${ }^{2}$ Department of Pediatric Hematology and Oncology, University of Health Sciences, Dr. \\ Abdurrahman Yurtaslan Ankara Oncology Training and Research Hospital; ${ }^{3}$ Department of Pediatric Infectious Diseases, University of \\ Health Sciences, Ankara Child Health and Diseases Hematology Oncology Training and Research Hospital; ${ }^{\circ}$ Department of Pathology, \\ University of Health Sciences, Ankara Child Health and Diseases Hematology Oncology Training and Research Hospital, Ankara, \\ Turkey.
}

\begin{abstract}
Background. Hemophagocytic lymphohistiocytosis (HLH) may precede malignancy, in particular lymphomas and leukemias. However, the causative factors, appropriate treatment and the prognosis of this association is not established.

Case. Herein, we present two patients, one with nodular sclerosing Hodgkin lymphoma (HL) and concomitant Epstein-Barr virus (EBV) infection, and the other with anaplastic large cell lymphoma (ALCL), presented as malignancy associated HLH.
\end{abstract}

Conclusion. In our patients, malignancy directed therapy was sufficient to treat HLH symptoms both at presentation and at recurrence in the second patient.

Key words: Hodgkin lymphoma, anaplastic large cell lymphoma, hemophagocytic lymphohistiocytosis.

Hemophagocytic lymphohistiocytosis (HLH) is a life-threatening, hyperinflammatory disorder demonstrated by activation of macrophages, cytotoxic $\mathrm{T}$ and natural killer (NK) cells. This uncontrolled immune response leading to macrophage activation and enhanced cytokinemia can be called a cytokine storm. ${ }^{1}$ In HLH diagnosis is based on refractory fever, hepatosplenomegaly, cytopenias, hypertriglyceridemia and/or hypofibrinogenemia, hemophagocytosis, low/ absent NK-cell-activity, hyperferritinemia, and high-soluble interleukin-2-receptor levels. ${ }^{2}$ It is classified into two subgroups; genetic (familial) and acquired (secondary). The familial HLH is characterized by a primary defect in cytotoxic

\footnotetext{
Tekin Aksu

tekinaksu@gmail.com
}

Received 18th October 2018, revised 3rd December 2018, 18th February 2019, accepted 28th May 2019. lymphocyte function (e.g., disrupted release of cytolytic granules) and autosomal recessive mode of inheritance. ${ }^{3}$

The diagnosis of HLH and especially primary or secondary HLH distinction may be challenging at the presentation of the patients. A search for underlying diseases should be performed for all patients, and initial treatment should not be delayed. Flow cytometric screening tests or molecular studies to detect the underlying genetic defects are available, but might be inconclusive due to insensitivity or unknown genetic defects. Familial HLH usually presents in infants or younger children where the trigger is often not apparent. ${ }^{3}$ Recurrent HLH and family history suggest primary HLH. Secondary HLH may develop due to several disorders, such as infections, rheumatologic diseases, and malign disorders. Additionally, malignancy-associated HLH (M-HLH) can be divided into two forms, where the malignancy 
triggers HLH via hyperinflammation and, persistent antigen stimulation by malignant cells or chemotherapy-associated HLH, where the infections or dysregulated immune system due to antineoplastic treatment provokes HLH. ${ }^{4,5}$ Also, hypomorphic mutations in familial HLH causing genes carried by adult patients are associated with late-onset HLH in the presence of viral infection or environmental stresses. ${ }^{6}$ Furthermore, a recent study suggested that monoallelic perforin gene (PRF1) mutations involved in lymphocyte survival and functional activity, may play a role in the development of lymphoid tumors. ${ }^{7}$ Although causative factors, appropriate treatment and the prognosis of this association is not established, the symptoms related to HLH often improve with treatment of the malignancy or HLH-directed therapy. Herein, we present two patients, one with Hodgkin lymphoma (HL), and the other with anaplastic large cell lymphoma (ALCL), presented with M-HLH.

\section{Case 1}

A previously healthy 12-year-old boy was referred to hospital with fever, abdominal mass and splenomegaly. His recent history revealed fever, fatigue and night sweats for the last month, and abdominal distention appeared one week before admission. In a local hospital, his hemoglobin $(\mathrm{Hb})$ level was found to be 7.1 $\mathrm{g} / \mathrm{dL}$, and he was transfused with erythrocyte suspension. Physical examination disclosed an abdominal mass $(10 \times 10 \mathrm{~cm})$ palpable on the umbilical area and his spleen was also palpable $10 \mathrm{~cm}$ below the left costal margin. There was no consanguinity between parents. Complete blood count $(\mathrm{CBC})$ revealed $\mathrm{Hb} 9 \mathrm{~g} / \mathrm{dl}$, white blood cell (WBC) count $6.6 \times 10^{9} / \mathrm{L}$, and platelet (Plt) count 199x10\% $/$ L. Peripheral smear, and bone marrow (BM) aspiration smear and biopsy were all normal. Abdominal and chest tomography disclosed mediastinal, and abdominal multiple conglomerated lymphadenopathies. Biopsy from abdominal lymphadenopathy was compatible with nodular sclerosing HL with Epstein-Barr virus (EBV) latent membrane protein (LMP) positivity. Before the initiation of chemotherapy, his fever continued, and petechia and ecchymosis were noticed on his trunk. At the time, a CBC showed $\mathrm{Hb} 7.7 \mathrm{~g} / \mathrm{dl}$, WBC count $3.4 \times 10^{9} / \mathrm{L}$, Plt count $21 \times 10^{9} / \mathrm{L}$, and a $\mathrm{BM}$ aspiration smear revealed hemophagocytic histiocytes (Fig. 1). His ferritin level was 1424 $\mathrm{ng} / \mathrm{ml}$, triglyceride (TG) level $181 \mathrm{mg} / \mathrm{dl}$, fibrinogen level $415 \mathrm{mg} / \mathrm{dl}$ and EBV polymerase chain reaction (EBV PCR) was 46.023 copies/ $\mathrm{ml}$. He had fever, splenomegaly, pancytopenia, hyperferritinemia, and hemophagocytosis which was compatible with HLH criteria reported by Henter et al. ${ }^{2}$ Finally, he was diagnosed with HLH secondary to HL and concomitant EBV infection. After one cycle of chemotherapy (ABVD; $25 \mathrm{mg} / \mathrm{m}^{2}$ doxorubicin, $9 \mathrm{mg} / \mathrm{m}^{2}$ bleomycin, $6 \mathrm{mg} / \mathrm{m}^{2}$ vinblastine, and $375 \mathrm{mg} / \mathrm{m}^{2}$ dacarbazine), the abdominal mass shrunk to about half of its size. His fever resolved and cytopenia improved, however histiocytes with hemophagocytosis persisted on a repeat $\mathrm{BM}$ examination. After the second course of chemotherapy, his mass could no longer be palpable on physical examination. BM aspiration revealed no hemophagocytosis and ferritin level was $252 \mathrm{ng} / \mathrm{ml}$, TG level $219 \mathrm{mg} / \mathrm{dl}$, fibrinogen level $252 \mathrm{mg} / \mathrm{dl}$ and EBV PCR were negative 45 days after the first examination. $\mathrm{He}$ is still in remission after 16 months. Informed

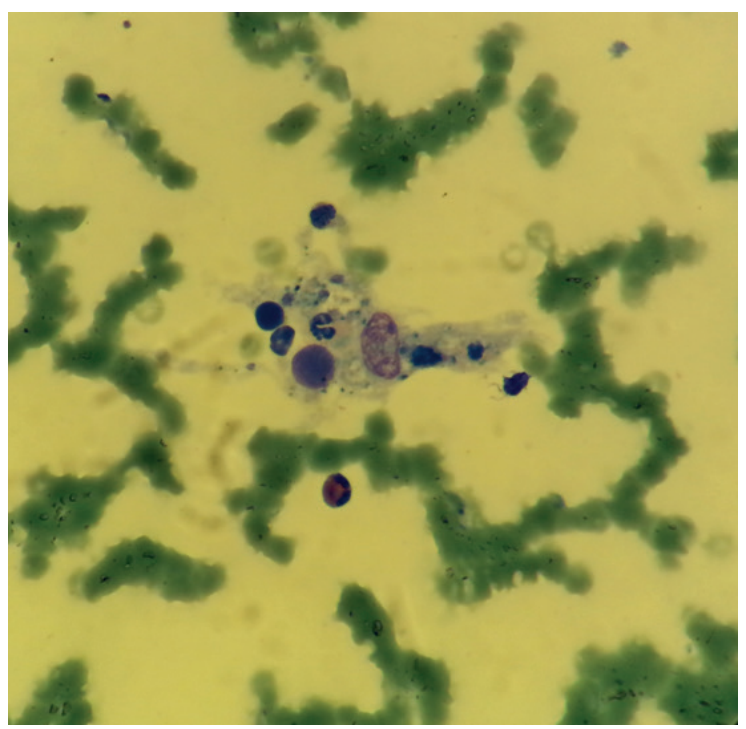

Fig. 1. Hemophagocytosis in bone marrow aspiration smear (magnification $\times 100$ ). 
consent was received from the patient and the family.

\section{Case 2}

A previously healthy 15-year-old boy presented with persistent fever $\left(>40 \mathrm{C}^{\circ}\right)$, fatigue, weight loss, skin rash and, hepatosplenomegaly. At admission, his CBC revealed $\mathrm{Hb} 7.9 \mathrm{~g} / \mathrm{dl}$, WBC count $0.3 \times 10^{9} / \mathrm{L}$, Plt count $52 \times 10^{9} / \mathrm{L}$. A peripheral blood smear showed leukopenia and thrombocytopenia without blasts. Alanine aminotransferase, aspartate aminotransferase, and gamma glutamyl transferase levels were 163, 431, and $404 \mathrm{U} / \mathrm{L}$, respectively. The direct bilirubin level was $2.71 \mathrm{mg} / \mathrm{dl}$. Serum ferritin was $3087 \mathrm{ng} / \mathrm{ml}$, TG level was $372 \mathrm{mg} / \mathrm{dl}$, and fibrinogen was low $(86 \mathrm{mg} / \mathrm{dl})$. BM aspiration smear showed marked hemophagocytosis, without blast, parasites or lipid-laden macrophages. EBV and cytomegalovirus (CMV) infections were excluded by PCR analysis. He had fever, splenomegaly, pancytopenia, hyperferritinemia, hypertriglyceridemia, hypofibrinogenemia, and hemophagocytosis which was compatible with HLH criteria reported by Henter et al. ${ }^{2}$ His chest X-ray disclosed pneumonitis without a clear etiology. Ultrasonography revealed multiple abdominal lymphadenomegaly and splenic infarct which was confirmed with computed tomography without portal or splenic venous thrombosis. Simultaneously, his blood cultures showed Candida albicans sensitive to amphotericin B. Considering his age; he was assumed as secondary HLH. High dose intravenous immunoglobulin (IVIG) $(2 \mathrm{~g} / \mathrm{kg})$ therapy was implemented without success. Since he had a refractory fever $\left(>40 \mathrm{C}^{\circ}\right)$, and septicemia, abdominal lymph node biopsy could not be performed. Due to our concern that steroids might impede the diagnosis of cancer, he received plasmapheresis for HLH for three consecutive days without clinical response. After completion of plasmapheresis, a nondiagnostic abdominal lymph node biopsy was attempted. Subsequently, high dose methylprednisolone (30 mg/kg, maximum dose $1 \mathrm{~g} /$ day) was initiated together with cyclosporine A $(5 \mathrm{mg} / \mathrm{kg} /$ day). This HLH-directed therapy attained clinical response at once, but it was not long-lasting. Fever, cytopenia, coagulopathy, and elevation of ferritin levels recurred five days after the initiation of steroid treatment. We studied NK cell-mediated cytotoxicity, and NK and T cell degranulation did not suggest a primary defect in cytotoxic lymphocyte function. Three weeks after admission and ten days under the steroid treatment, he developed a maculopapular rash on his trunk. A skin biopsy showed anaplastic large cell kinase positive- ALCL. Therefore, he was diagnosed with HLH due to stage III ALCL four weeks after admission to our hospital. He received one course of dexamethasone (10 $\mathrm{mg} / \mathrm{m}^{2} /$ day, five days), methotrexate (3 gr/ $\left.\mathrm{m}^{2}\right)$, ifosfamide $\left(800 \mathrm{mg} / \mathrm{m}^{2} /\right.$ day, five days), cytarabine $\left(150 \mathrm{mg} / \mathrm{m}^{2} /\right.$ dose, four doses), and etoposide (100 mg/m²/day, two days). After the treatment, his fever resolved, and cutaneous lesions disappeared. He took this chemotherapy course with alternating dexamethasone (10 $\mathrm{mg} / \mathrm{m}^{2} /$ day, five days), methotrexate $\left(3 \mathrm{gr} / \mathrm{m}^{2}\right)$, cyclophosphamide (200 mg/m²/day, five days), doxorubicin ( $25 \mathrm{mg} / \mathrm{m}^{2} /$ day, two days) for a total of six cycles. At the end of chemotherapy, he was in remission, however, two months after the cessation of therapy cutaneous lesions reappeared. Pathological examination of cutaneous lesions revealed ALCL. Interestingly, at the time of relapse, he had fever, cytopenia, hyperferritinemia, lymphadenopathies and splenomegaly, and hemophagocytosis in BM that we assumed recurrence of M-HLH. He is now in remission after three courses of chemotherapy and waiting for hematopoietic stem cell transplantation. Informed consent was received from the patient and the family.

\section{Discussion}

Existing evidence suggests that secondary HLH in patients who have cancer is multifactorial. The infections, severe inflammation triggered by malignancy, and loss of immune homeostasis due to anti-neoplastic treatment may lead to secondary HLH. Additionally hypomorphic 
HLH causing gene mutations may act as a primary cofactor of Malignancy-associated HLH (M-HLH) in these patients. ${ }^{5}$ Malignancy induced HLH is reported to have an incidence of $1.2 \%$, and in particular, associated with lymphomas and leukemias. ${ }^{4,8}$ Notably $\mathrm{T}$ cell and NK cell lymphomas cause HLH due to strong relation of these tumors with EBV infection. ${ }^{9}$ A recent manuscript concerning pediatric and adolescent patients reported that malignancy was suspected in $8.4 \%$ of patients with HLH. Also, they found that most of the HLH presented before at the onset or of certain malignancies, mainly ALCL and HL as in our patients. Also, they found the median age was 12 years for malignancy-triggered $\mathrm{HLH}$, and 5.5 years for chemotherapy-related HLH. ${ }^{4}$ In our report, both of the patients were adolescents and HLH presented at the onset of HL in one of them, and before ALCL in the other. On the other hand, Strenger et al. ${ }^{8}$ described 22 patients with M-HLH in which most of the patients developed HLH during hemato-/oncologic treatment.

Hodgkin lymphoma associated with HLH has been reported in a few case reports and case series. ${ }^{9-18}$ Menard et al. ${ }^{14}$ revealed EBV positivity in tumor cells via EBER and/or LMP-1 in 32 of the 34 adult patients with HLH associated with HL. They claimed that high expression of EBV LMP-1 in tumor cells might induce Th1 cells to produce large amounts of cytokines and initiate HLH process. ${ }^{14}$ In our first patient, it seems EBV was a co-trigger contributing to cytokinemia and the development of M-HLH. HLH has been reported as an initial presentation of HL and associated with BM involvement possibly by inducing a cytokine storm. ${ }^{9-15,17}$ However, our patient developed HLH after the initial presentation of HL, but before chemotherapy and without BM involvement.

Anaplastic large cell lymphoma, one of the most common pediatric large-cell lymphomas included in the mature T-cell lymphoma group usually admits with extranodal involvement, skin, and systemic symptoms. ${ }^{19}$ It may also present with $\mathrm{HLH} .{ }^{20}$ Some clinical findings of ALCL are similar to HLH symptoms including fever, lymphadenopathy, skin rash, and hemophagocytosis. Studies have shown that proinflammatory cytokines which were elevated and possibly produced by malignant cells, may play a role in the clinical picture. ${ }^{1,21}$ Additionally, Ciambotti et al. ${ }^{7}$ suggest that mutations of PRF1 in ALCL patients were missense mutations that impaired perforin function weakly, which were not enough to cause an HLH attack. Since our patient's T- and NK-cell functions were normal, we did not analyze PRF1 mutations in our patients. However, at the time of relapse HLH also recurred that may occur due to the possible PRF1 mutation. Also, Candida infection could be a co-trigger or co-infection for M-HLH in the patient. In our patients, treatment of malignancy and infection was sufficient to treat HLH symptoms both at presentation and at recurrence in the second patient.

In this report both of the patients met the HLH criteria reported by Henter et al. ${ }^{2}$ However, the widely used HLH-2004 criteria may not be sufficient to diagnose HLH in a patient who also has active malignancy, as some features may be related to the malignancy itself. Daver et al. ${ }^{5}$ proposed a schema containing 18 variables for adult M-HLH that incorporates a more accessible physical examination and laboratory variables. They claimed that patients who have any 5 of these 18 variables could be M-HLH. Despite the limitations, particularly for M-HLH, the HLH-2004 criteria are still the widely accepted definition.

In conclusion, we suggested secondary HLH for patients due to the late-onset presentation, normal cytotoxic lymphocyte function, and no recurrent disease or family history. HLH is an infrequent complication of HL and ALCL in children and may mask the primary tumor. Infections may be co-triggers for M-HLH, and should be treated expeditiously. Treatment should be directed to the malignant condition instead of HLH. 


\section{REFERENCES}

1. Shimada A, Kato M, Tamura K, et al. Hemophagocytic lymphohistiocytosis associated with uncontrolled inflammatory cytokinemia and chemokinemia was caused by systemic anaplastic large cell lymphoma: a case report and review of the literature. J Pediatr Hematol Oncol 2008; 30: 785-787.

2. Henter JI, Horne A, Arico M, et al. HLH-2004: diagnostic and therapeutic guidelines for hemophagocytic lymphohistiocytosis. Pediatr Blood Cancer 2007; 48: 124-131.

3. Jordan MB, Allen CE, Weitzman S, Filipovich $\mathrm{AH}$, Mcclain LK. How I treat hemophagocytic lymphohistiocytosis. Blood 2011; 118: 4041-4052.

4. Lehmberg K, Sprekels B, Nichols KE, et al. Malignancy-associated haemophagocytic lymphohistiocytosis in children and adolescents. $\mathrm{Br}$ J Haematol 2015; 170: 539-549.

5. Daver N, McClain K, Allen CE, et al. A consensus review on malignancy-associated hemophagocytic lymphohistiocytosis in adults. Cancer 2017; 123: 3229-3240.

6. Zhang $\mathrm{K}$, Jordan MB, Marsh RA, et al. Hypomorphic mutations in $\mathrm{PRF}_{1}, \mathrm{MUNC}_{13-4^{\prime}}$ and $\mathrm{STXBP}_{2}$ are associated with adult-onset familial HLH. Blood 2011; 118: 5794-5798.

7. Ciambotti B, Mussolin L, d'Amore ESG, et al. Monoallelic mutations of the perforin gene may represent a predisposing factor to childhood anaplastic large cell lymphoma. J Pediatr Hematol Oncol 2014; 36: e359-e365.

8. Strenger V, Merth G, Lackner H, et al. Malignancy and chemotherapy induced haemophagocytic lymphohistiocytosis in children and adolescents-a single centre experience of 20 years. Ann Hematol 2018; 97: 989-998.

9. Hagihara M, Inoue M, Hua J, Iwaki Y. Lymphocytedepleted Hodgkin lymphoma complicating hemophagocytic lymphohistiocytosis as an initial manifestation: a case report and review of the literature. Intern Med 2012; 51: 3067-3072.

10. Abaza H, Ben Chaabene A, Sallami S, Boussen H, Rahal K, Guemira F. Hemophagocytic syndrome associated to a Hodgkin lymphoma. Tunis Med 2015; 93: 399-400.
11. Chan K, Behling E, Strayer DS, Kocher WS, Dessain SK. Prolonged hemophagocytic lymphohistiocytosis syndrome as an initial presentation of Hodgkin lymphoma: a case report. J Med Case Rep 2008; 2: 367.

12. Dominguez-Munoz MA, Morales-Camacho RM, Prats-Martin C, et al. Unusual co-occurrence of Hodgkin lymphoma and hemophagocytic lymphohistiocytosis in a bone marrow aspirate. Ann Hematol 2016; 95: 1019-1021.

13. Ichikawa S, Takahashi T, Katsushima H, Fukuhara N, Ichinohasama R, Harigae H. Advanced lymphocyterich classical Hodgkin lymphoma complicated with fatal hemophagocytic syndrome. Intern Med 2016; 55: 191-196.

14. Menard F, Besson C, Rince P, et al. Hodgkin lymphoma-associated hemophagocytic syndrome: a disorder strongly correlated with Epstein-Barr virus. Clin Infect Dis 2008; 47: 531-534.

15. Morita Y, Kenzaka T, Yoshimoto H, Ohno N. Hodgkin's lymphoma preceded by haemophagocytic lymphohistiocytosis. BMJ Case Rep 2013; 2013.

16. Roe C, Bennett J, Zhang L, et al. Hemophagocytic lymphohistiocytosis in malignant hematology: uncommon but should not be forgotten? Clin Lymphoma Myeloma Leuk 2015; 15: S147-S150.

17. Tatara R, Sato M, Fujiwara SI, et al. Hemoperfusion for Hodgkin lymphoma-associated hemophagocytic lymphohistiocytosis. Intern Med 2014; 53: 2365-2368.

18. Celkan T, Berrak S, Kazanci E, et al. Malignancyassociated hemophagocytic lymphohistiocytosis in pediatric cases: a multicenter study from Turkey. Turk J Pediatr 2009; 51: 207-213.

19. Swerdlow SH, Campo E, Pileri SA, et al. The 2016 revision of the World Health Organization classification of lymphoid neoplasms. Blood 2016 127: 2375-2390.

20. Li W, Repnikova E. ALK-positive anaplastic largecell lymphoma with marked leukemoid reaction and hemophagocytic lymphohistiocytosis. Blood 2016; 127: 2041.

21. Al-Hashmi I, Decoteau J, Gruss HJ, et al. Establishment of a cytokine-producing anaplastic large-cell lymphoma cell line containing the $t(2 ; 5)$ translocation: potential role of cytokines in clinical manifestations. Leuk Lymphoma 2001; 40: 599-611. 